\title{
Understanding risk of thrombosis with thrombocytopenia syndrome after Ad26.COV2.S vaccination
}

\author{
Prathit A. Kulkarni $(\bowtie)^{1,2}$, Vinay Prasad ${ }^{3}$ \\ ${ }^{1}$ Infectious Diseases Section, Department of Medicine, Baylor College of Medicine, Houston, TX 77030, USA; ${ }^{2}$ Medical Care Line, Michael \\ E. DeBakey Veterans Affairs Medical Center, Houston, TX 77030, USA; ${ }^{3}$ Department of Epidemiology and Biostatistics, School of Medicine, \\ University of California-San Francisco, San Francisco, CA 94143, USA
}

(C) Higher Education Press 2021

On April 13, 2021, the US Centers for Disease Control and Prevention (CDC) and the US Food and Drug Administration (FDA) recommended a pause in administration of the Ad26.COV2.S (Johnson \& Johnson [Janssen]) coronavirus disease 2019 (COVID-19) vaccine for all persons [1]. At that time, approximately 6.8 million doses of vaccine had been administered around the United States. The pause was prompted by 6 cases of cerebral venous sinus thrombosis (CVST) in combination with thrombocytopenia, a condition whose nomenclature is in flux but which we will refer to as thrombosis with thrombocytopenia syndrome (TTS), as used by CDC in describing the condition [2]. All patients were women younger than 50 years. Of note, 2 of 6 patients also had splanchnic and portal vein thrombosis. As of May 12, 2021, CDC's working case definition for TTS after COVID-19 vaccination divides the condition into two tiers [3]. A case of tier 1 TTS is defined as: (1) thrombosis in an unusual location; and (2) thrombocytopenia with a platelet count $<150000 /$ microliter. A positive heparin-platelet factor 4 (PF4) antibody is supportive but not required. A tier 2 TTS case is defined as: (1) thrombosis only in a common location (such as deep vein thrombosis or pulmonary embolism); (2) thrombocytopenia with a platelet count $<150000 /$ microliter; and (3) positive heparin-PF4 antibody.

Many in the scientific community were concerned that this pause was misguided. These concerns were understandable given the ongoing COVID-19 pandemic. Some stated that the risk of "clot" after receiving the Johnson \& Johnson vaccine was less than 1 in 1 million. By looking

Received May 27, 2021; accepted August 28, 2021

Correspondence: Prathit A. Kulkarni, pakulkar@bcm.edu at only raw numbers (6 cases out of approximately 6.8 million doses of vaccine given), this is technically true. However, there are several unique features of the reported adverse events that supported the initial pause and need for additional inquiry.

First, the location of the clotting was highly unusual. CVST is an uncommon diagnosis, with an approximate annual incidence rate of 5 per million in the general population [4]. Treating this clot is challenging because of the clinical consequences and potential concurrent hemorrhage. Current practice is to continue anticoagulation, though evidence remains scant [4]. Therefore, comparisons of TTS resulting in CVST to the baseline incidence rate of all cases of typical venous thromboembolism (VTE), such as those in the legs associated with oral contraceptive pills (OCPs), are misleading. It is also likely the case that a typical treatment for CVST, heparin, might not be usable in these vaccine-related cases, thereby making clinical management even more challenging.

Second, all 6 reported cases were associated with thrombocytopenia. This was immediately and correctly recognized as highly unusual, as there are few conditions, such as heparin-induced thrombocytopenia and thrombosis (HITT) and thrombotic thrombocytopenic purpura, which have the unusual association of low platelets and clotting. HITT, for example, is a state of runaway platelet activation, which can result in arterial and venous thrombosis. In this context, analogies to typical VTE again might not be appropriate.

Third, all 6 cases of CVST initially reported in the United States were in women aged $<50$ years. These initial data suggested that this was the group at highest risk for potential TTS after receipt of the Johnson \& Johnson COVID-19 vaccine, not simply all comers. As such, given the unusual clinical presentation of a novel syndrome with 
potentially dire and irreversible consequences in a young, healthy subpopulation of vaccine recipients, a pause appears to have been reasonable.

Importantly, the relevant comparison for understanding risk of TTS associated with the Johnson \& Johnson COVID-19 vaccine is not the baseline rate of all VTE in the general population or even the baseline rate of CVST in women less than age 50 . The most accurate comparison of risk is the baseline incidence rate of CVST associated with thrombocytopenia in women aged $<50$ years of age who have not received a heparin product. In a study that evaluated a cohort of 865 patients with CVST from 7 countries around the world from 1987 through March 2018, 73 (8.4\%) were found to have concomitant thrombocytopenia [5]. The cause of thrombocytopenia was known in $64 \%$ of these patients. One patient was found to have positive heparin-PF4 antibody diagnosed as part of routine care. This patient had received lowmolecular weight heparin as part of her care. Among 93 frozen plasma samples subsequently evaluated for presence of heparin-PF4 antibodies, none were found to be positive. Overall, the incidence of CVST occurring in conjunction with thrombocytopenia mediated via heparinPF4 antibodies was exceedingly rare in this cohort of patients with CVST from the general population $(0.1 \%$ of patients), and in fact only occurred in a patient who had received a heparin product. Therefore, risk of CVST with potentially antibody-mediated thrombocytopenia related to the Johnson \& Johnson COVID-19 vaccine is, by definition, excess above an almost negligible baseline.

After the initial pause in national administration of the Johnson \& Johnson vaccine on April 13, 2021, an additional 9 cases of TTS were publicly reported. The total of 15 cases, 12 of which included CVST, were discussed at a meeting of the US Advisory Committee on Immunization Practices (ACIP) on April 23, 2021 [6]. Thirteen of the 15 cases were in women younger than age 50 . With approximately 1.9 million doses of vaccine given in this age group, the reporting rate of TTS was noted to be 7.0 per million. Simultaneously, the mortality rate of COVID-19 for women less than age 50 was reported to be less than 1 in 100000 . Therefore, the risk of developing TTS after administration of the Johnson \& Johnson vaccine was roughly similar to the risk of mortality from COVID-19 in the same age and sex demographic. As described during the meeting, for women aged 18-49 years, for every 1 million doses of vaccine given with current risk of exposure to severe acute respiratory syndrome coronavirus 2 (SARS-CoV-2) in the United States, there would be 13 expected cases of TTS, and 12 deaths related to COVID-19 would be prevented.

With regard to the potential pathophysiological basis for vaccine-related TTS, a description of the first 12 patients in the United States with the condition was published in late April 2021 [7]. All patients were women between the ages of 18 and 60 . Eleven of 12 patients were younger than age 50 , and $8 / 12$ additionally had thromboses in locations other than the cerebral circulation. A total of 11 patients had positive heparin-PF4 antibodies, and 1 patient did not have the testing performed. However, 8/11 had negative functional platelet antibody results with 6 having undergone serotonin release assay testing only and 2 having undergone both serotonin release assay testing and latex immunoturbidimetric assay testing. Other thrombophilia testing was negative in these 11 patients.

Just prior to the initial reports of TTS occurring after vaccination with the Johnson \& Johnson COVID-19 vaccine, descriptions of thrombotic thrombocytopenia related to the ChAdOx1 nCov-19 (AstraZeneca) COVID19 vaccine (which also uses Adenovirus-vector vaccine technology) were published [8]. The initial report of 11 patients from Germany and Austria suggested that the clotting observed in a condition termed vaccine-induced immune thrombotic thrombocytopenia (VITT) - and remarkably similar in description to cases of TTS described in the United States - might also be mediated by antibody-driven activation of PF-4. This would make the condition most similar to HITT. A report simultaneously published on the same day described 5 additional patients from Norway, all younger than age 60, with CVST and thrombocytopenia [9]. All patients had strongly positive anti-heparin-PF4 antibodies. Another report of 22 patients with acute thrombosis in unusual locations along with thrombocytopenia confirmed the presence of anti-heparin-PF4 antibodies as well [10]. In contrast to the series of patients described in the United States, the vast majority of patients described in these European reports had positive functional platelet antibody assays. As described by See et al., the reason for this difference could be due to false-negative test results or potentially different types of functional platelet antibody tests that were used in different settings [7]. Indeed, a detailed discussion of anti-PF4 antibodies describes how rapid tests typically used for diagnosis of HITT might not be positive in patients with VITT [11]. In addition, standard serotonin release assays that do not include the presence of PF4 were often negative but turned positive when PF4 was introduced into the mixture. Specifically, the authors of this study related to PF4 immunoassays recommend use of a "sensitive, quantitative, immunologic test" for diagnosis of VITT [11].

To note, two other COVID-19 vaccines which utilize Adenovirus-vector technology have received authorization in different parts of the world but not in the United States. These are the Gam-COVID-Vac (Sputnik V) vaccine from Russia, which uses 2 components, a recombinant adenovirus type 26 vector and a recombinant adenovirus type 5 vector in a heterologous prime-boost fashion, and the CanSinoBIO vaccine from China, which also uses an adenovirus type 5 vector. However, there have not been to 
date any public reports of serious episodes of thrombosis associated with either of these vaccines. Additionally, there is one other vaccine that currently has approval for use in Europe which utilizes Adenovirus-vector technology, Ad26.ZEBOV, also a Johnson \& Johnson product incorporating adenovirus type 26. This vaccine, for prevention of Ebola virus disease, has not been reported in the literature to be linked to serious clotting disorders.

By the time of a follow-up meeting of ACIP on May 12, 2021, a total of 28 cases of TTS had been confirmed by CDC, with 25/28 cases being in the Tier 1 category [3]. Among the 28 cases, 19 cases included CVST. At a subsequent ACIP meeting on July 22, 2021, the number of cases of confirmed reports of TTS had risen to 38 , with $28 / 38$ cases occurring in females [12]. The reporting rate of TTS among females aged 30-49 years was 8.8 per million; in women aged 18-29 years, it was 4.2 per million. As noted during that meeting, based upon average exposure risk and hospitalization rates in the United States from June 19, 2021, for every million doses of Johnson \& Johnson vaccine given, for women aged $18-29,4-5$ cases of TTS would be expected, and 5 deaths would be prevented. For women aged $30-49$, a total of 8-10 cases of TTS would be expected, and 20 deaths would be averted.

A crucial point in a risk-benefit analysis is that risk of acquiring SARS-CoV-2 infection is not static, and also differs from one individual to another. For example, if someone has an occupation and lifestyle that allow them to avoid interaction with others and they adhere strictly to recommended public health guidance, their risk of acquiring SARS-CoV-2 infection might be extremely low. Risk is also affected by viral transmission rates, which are constantly changing in different geographical areas. In addition, mRNA vaccine options are now widely available throughout the United States; therefore, it is not the case that no form of protection would be available for patients if they opted not to receive the Johnson \& Johnson vaccine. It is important to note that population-based recommendations in countries around the world where vaccine supply could be different would have a different risk-benefit analysis.

The current approach for use of the Johnson \& Johnson COVID-19 vaccine in the United States is that it is recommended under emergency use authorization (EUA) for all persons greater than age 18. However, EUA was amended on April 23, 2021, and now includes a warning label regarding the possibility of TTS related to the vaccine. To note, certain countries in Europe have opted to restrict use of Adenovirus-vector vaccines to certain age groups only. As an example, in May 2021, the Joint Committee on Vaccination and Immunisation in the UK advised the following: "in addition to those aged under 30, unvaccinated adults aged 30-39 years who are not in a clinical priority group at higher risk of severe COVID-19 disease, should preferentially be offered an alternative to the AstraZeneca COVID-19 (AZD1222) vaccine" assuming no delay in vaccination occurs [13]. Therefore, clinicians might have continued concerns about recommending or administering the Johnson \& Johnson vaccine to women younger than age 50, particularly when alternatives (mRNA vaccines) are now widely available. As noted by the ACIP Work Group on benefits and risks of COVID-19 vaccination on July 22, 2021, reported adverse events from vaccination are "potentially serious and should be transparently communicated with the public" [14]. The work group also noted the "importance of patient choice in vaccine product" and that "patients and providers should be aware of both the benefits and risks of COVID-19 vaccination when choosing a vaccine product." The authors agree with these sentiments. Ultimately, a clear articulation of risks and benefits, and an individual discussion with each patient, will be needed as clinicians help patients select a vaccine product.

\section{Compliance with ethics guidelines}

Prathit A. Kulkarni discloses the following: Research funding: Vessel Health, Inc.; Michael E. DeBakey VA Medical Center Research Service Line; Department of Veterans Affairs Office of Research and Development; Speaking: National AIDS Education \& Training Center.

Vinay Prasad discloses the following: Research funding: Arnold Ventures; Royalties: Johns Hopkins Press, Medscape, MedPage Today; Consulting: UnitedHealthcare; Speaking: Evicore, New Century Health; Other: Patreon backers for Plenary Session podcast.

This manuscript did not involve reporting of primary data obtained from studies of human or animal subjects. It is solely a comment manuscript.

\section{References}

1. U.S. Centers for Disease Control \& Prevention. Joint CDC and FDA statement on Johnson \& Johnson COVID-19 vaccine. Press release on April 13, 2021. 2021. https://www.cdc.gov/media/releases/2021/ s0413-JJ-vaccine.html (accessed April 24, 2021)

2. U.S. Centers for Disease Control \& Prevention. Health Alert Network Advisory on April 13, 2021. 2021. https://emergency.cdc. gov/han/2021/han00442.asp (accessed April 24, 2021)

3. U.S. Centers for Disease Control \& Prevention. ACIP presentation slides: May 12, 2021 meeting. 2021. https://www.cdc.gov/vaccines/ acip/meetings/downloads/slides-2021-05-12/07-COVID-Shimabukuro-508.pdf (accessed August 2, 2021)

4. Saposnik G, Barinagarrementeria F, Brown RD Jr, Bushnell CD, Cucchiara B, Cushman M, deVeber G, Ferro JM, Tsai FY. Diagnosis and management of cerebral venous thrombosis: a statement for healthcare professionals from the American Heart Association/ American Stroke Association. Stroke 2011; 42(4): 1158-1192

5. Sánchez van Kammen M, Heldner MR, Brodard J, Scutelnic A, Silvis S, Schroeder V, Kremer Hovinga JA, Middeldorp S, Levi M, 
Hiltunen S, Lindgren E, Mansour M, Arauz A, Barboza MA, Zuurbier SM, Aguiar de Sousa D, Ferro JM, Fischer U, Field TS, Jood K, Tatlisumak T, Putaala J, Arnold M, Coutinho JM. Frequency of thrombocytopenia and platelet factor 4/heparin antibodies in patients with cerebral venous sinus thrombosis prior to the COVID-19 pandemic. JAMA 2021; 326(4): 332-338

6. U.S. Centers for Disease Control \& Prevention. ACIP presentation slides: April 23, 2021 meeting. 2021. https://www.cdc.gov/ vaccines/acip/meetings/slides-2021-04-23.html (accessed April 24, 2021)

7. See I, Su JR, Lale A, Woo EJ, Guh AY, Shimabukuro TT, Streiff MB, Rao AK, Wheeler AP, Beavers SF, Durbin AP, Edwards K, Miller E, Harrington TA, Mba-Jonas A, Nair N, Nguyen DT, Talaat KR, Urrutia VC, Walker SC, Creech CB, Clark TA, DeStefano F, Broder KR. US case reports of cerebral venous sinus thrombosis with thrombocytopenia after Ad26.COV2.S vaccination, March 2 to April 21, 2021. JAMA 2021; 325(24): 2448-2456

8. Greinacher A, Thiele T, Warkentin TE, Weisser K, Kyrle PA, Eichinger S. Thrombotic thrombocytopenia after ChAdOx1 nCov19 vaccination. N Engl J Med 2021; 384(22): 2092-2101

9. Schultz NH, Sárvoll IH, Michelsen AE, Munthe LA, Lund-Johansen F, Ahlen MT, Wiedmann M, Aamodt AH, Skattør TH, Tjønnfjord GE, Holme PA. Thrombosis and thrombocytopenia after ChAdOx1 nCov-19 vaccination. N Engl J Med 2021; 384(22): 2124-2130
10. Scully M, Singh D, Lown R, Poles A, Solomon T, Levi M, Goldblatt D, Kotoucek P, Thomas W, Lester W. Pathologic antibodies to platelet factor 4 after ChAdOx1 nCoV-19. N Engl J Med 2021; 384 (23): 2202-2211

11. Vayne C, Rollin J, Gruel Y, Pouplard C, Galinat H, Huet O, Mémier V, Geeraerts T, Marlu R, Pernod G, Mourey G, Fournel A, Cordonnier C, Susen S. PF4 immunoassays in vaccine-induced thrombotic thrombocytopenia. N Engl J Med 2021; 385(4): 376378

12. U.S. Centers for Disease Control \& Prevention. ACIP presentation slides: July 22, 2021 meeting. 2021. https://www.cdc.gov/vaccines/ acip/meetings/downloads/slides-2021-07/05-COVID-Rosenblum508.pdf (accessed August 2, 2021)

13. United Kingdom Joint Committee on Vaccination and Immunisation. Use of the AstraZeneca COVID-19 (AZD1222) vaccine: Updated JCVI statement, 7 May 2021. 2021. https://www.gov.uk/ government/publications/use-of-the-astrazeneca-covid-19-vaccinejcvi-statement-7-may-2021/use-of-the-astrazeneca-covid-19azd1222-vaccine-updated-jcvi-statement-7-may-2021 (accessed August 2, 2021)

14. U.S. Centers for Disease Control \& Prevention. ACIP presentation slides: July 22, 2021 meeting. 2021. https://www.cdc.gov/vaccines/ acip/meetings/downloads/slides-2021-07/06-COVID-Mbaeyi-508. pdf (accessed August 2, 2021) 